\title{
MAXIMAL SUBLATTICES AND FRATTINI SUBLATTICES OF BOUNDED LATTICES
}

\author{
M. E. ADAMS, RALPH FREESE, J. B. NATION and JÜRG SCHMID
}

(Received 15 November 1995; revised 31 January 1997)

Communicated by B. A. Davey

\begin{abstract}
We investigate the number and size of the maximal sublattices of a finite lattice. For any positive integer $k$, there is a finite lattice $\mathbf{L}$ with more that $|L|^{k}$ sublattices. On the other hand, there are arbitrary large finite lattices which contain a maximal sublattice with only 14 elements. It is shown that every finite bounded lattice is isomorphic to the Frattini sublattice (the intersection of all maximal sublattices) of a finite bounded lattice.
\end{abstract}

1991 Mathematics subject classification (Amer. Math. Soc.): primary 06B05, 06B20.

The Frattini sublattice $\Phi(\mathbf{L})$ of a lattice $\mathbf{L}$ is the intersection of all maximal (proper) sublattices of $\mathbf{L}$. In this note we are concerned with the following problems:

(1) How many maximal sublattices can a finite lattice of size $n$ have?

(2) How small can a maximal sublattice of a finite lattice be?

(3) For which lattice varieties $\mathscr{V}$ is it true that every finite non-trivial lattice $\mathbf{L} \in \mathscr{V}$ is isomorphic to $\Phi\left(\mathbf{L}^{\prime}\right)$ for some finite lattice $\mathbf{L}^{\prime} \in \mathscr{V}$ ?

We will address these questions in order, and provide good partial answers, especially for finite lattices which are bounded homomorphic images of a free lattice. Recall that a finite lattice is bounded if and only if it can be obtained from the one element lattice by a sequence of applications of Alan Day's doubling construction for intervals. In particular, finite distributive lattices are bounded. On the other hand, we do not have a complete solution for any of the above problems.

The main results of this paper can be summarized as follows.

(1a) For any $k>0$, there exists a finite lattice $\mathbf{L}$ which has more than $|L|^{k}$ maximal sublattices.

The authors were supported in part by NSF Grants DMS 95-00752 (Freese) and DMS 94-0051 1 (Nation).

(C) 1997 Australian Mathematical Society 0263-6115/97 \$A2.00+0.00 
(lb) A finite bounded lattice $\mathbf{L}$ has at most $|L|$ maximal sublattices.

(2a) There exist arbitrarily large finite (or even countably infinite) lattices with a maximal sublattice isomorphic to the five element lattice $\mathbf{M}_{3}$.

(2b) For any $\varepsilon>0$, there exists a finite bounded lattice $\mathbf{L}$ with a maximal sublattice S such that $|S|<\varepsilon|L|$.

(3a) There exist infinitely many lattice varieties $\mathscr{V}$ such that every finite non-trivial lattice $\mathbf{L} \in \mathscr{V}$ is isomorphic to $\Phi\left(\mathbf{L}^{\prime}\right)$ for some finite lattice $\mathbf{L}^{\prime} \in \mathscr{V}$.

(3b) Every finite bounded lattice $\mathbf{L}$ can be represented as $\Phi(\mathbf{K})$ for some finite bounded lattice $\mathbf{K}$ (not necessarily in $\mathscr{V}(\mathbf{L})$ ).

\section{Preliminaries}

We need to recall some basic results and terminology for semidistributive and bounded lattices. There is a relatively complete treatment of bounded lattices in the book [7], to which we shall refer. The semidistributive laws are given by

$$
\begin{array}{llll}
\left(\mathrm{SD}_{\vee}\right) & u=a \vee b=a \vee c & \text { implies } & u=a \vee(b \wedge c) \\
\left(\mathrm{SD}_{\wedge}\right) & v=a \wedge b=a \wedge c & \text { implies } & v=a \wedge(b \vee c) .
\end{array}
$$

For a finite lattice, $\left(\mathrm{SD}_{\vee}\right)$ is equivalent to the property that every element has a canonical join representation, that is, for every $a \in L$ there exists $C \subseteq \mathrm{J}(\mathbf{L})$ such that $a=\bigvee C$ irredundantly, and $a=\bigvee B$ implies that for every $c \in C$ there exists $b \in B$ with $c \leq b$; see [7, Theorem 2.24]. The elements of $C$ are called the canonical joinands of $a$, and we write $C=\mathrm{CJ}(a)$. Of course, $\left(\mathrm{SD}_{\wedge}\right)$ is equivalent to the dual property, and the corresponding elements are called the canonical meetands of $a$, and denoted by $\mathrm{CM}(a)$.

We will use a basic fact about semidistributive lattices. A finite lattice $\mathbf{L}$ satisfies $\left(\mathrm{SD}_{\wedge}\right)$ if and only if for each join irreducible element $p$ of $\mathbf{L}$ there is a unique element $\kappa(p)$ in $\mathbf{L}$ which is maximal with respect to $x \geq p_{*}$ and $x \nsupseteq p$, where $p_{*}$ denotes the unique lower cover of $p$. Moreover, the elements $\kappa(p)$ are precisely the meet irreducible elements of $\mathbf{L}$. (See [7, Theorem 2.54].)

Let $\mathrm{L}$ be a finite lattice and $p \in L$. Any set $Q$ such that $p \leq \bigvee Q$ is called a join cover of $p$. A join cover is non-trivial if $p \not \leq q$ for all $q \in Q$. It is minimal if $Q \subseteq \mathbf{J}(\mathbf{L})$ and no element of $Q$ can be deleted or replaced by a smaller element.

We can define subsets $\mathrm{D}_{k}(\mathbf{L}) \subseteq L$ for $k \in \omega$ as follows. $\mathrm{D}_{0}(\mathbf{L})$ consists of the join prime elements of $\mathbf{L}$, that is, those elements with no non-trivial join cover. Inductively, an element $p$ is in $D_{k+1}(\mathbf{L})$ if every minimal non-trivial join cover of $p$ is contained in $\mathrm{D}_{k}(\mathbf{L})$. Note

$$
\mathrm{D}_{0}(\mathbf{L}) \subseteq \mathrm{D}_{1}(\mathbf{L}) \subseteq \mathrm{D}_{2}(\mathbf{L}) \subseteq \cdots
$$


The finite lattice $\mathbf{L}$ is bounded (in the sense of McKenzie [11]) if it is semidistributive and $\mathrm{D}_{k}(\mathbf{L})=L$ for some $k$ (combining Theorems 2.13 and 2.64 of [7]). Equivalently, $\mathbf{L}$ is bounded if it can be obtained from a one element lattice by a sequence of doublings of intervals; this result of Day [5] and [6] is Corollary 2.44 of [7]. The D-rank of an element $x$ in a bounded lattice is the least $k$ such that $x \in \mathrm{D}_{k}(\mathbf{L})$.

A straightforward argument shows that if $\mathrm{J}(\mathbf{L}) \subseteq \mathrm{D}_{n}(\mathbf{L})$, then $\mathrm{D}_{n+1}(\mathbf{L})=L$; see [7, Lemma 2.5]. The class of finite bounded lattices does not form a variety, but for a fixed $n$, the finite semidistributive lattices such that $\mathrm{J}(\mathbf{L}) \subseteq \mathrm{D}_{n}(\mathbf{L})$ are the finite members of a locally finite variety $\mathscr{B}_{n}$, described in more detail in Nation [12] (cf. [7, Corollary 2.17]). For example, $\mathscr{B}_{0}$ is the variety of distributive lattices, while the pentagon $\mathbf{N}_{5}$ is contained in $\mathscr{B}_{1}$. Clearly, a finite lattice is bounded if and only if it is in $\mathscr{B}_{n}$ for some $n$.

Throughout we will use the convention that algebras or relational structures are denoted by bold face capitals, for example $\mathbf{L}$, while their carrier sets are denoted by the corresponding plain letters, for example $L$.

\section{The number of maximal sublattices}

A relatively free lattice with $n$ generators has exactly $n$ maximal sublattices, each obtained by removing one of the generators (which are doubly irreducible). Thus there exist arbitrarily large finite lattices with only 3 maximal sublattices.

So let us turn our attention to finding finite lattices with lots of maximal sublattices. In general, an algebra of cardinality $n$ can have at most $\left(\begin{array}{c}n \\ \lfloor n / 2\rfloor\end{array}\right)$ maximal subalgebras, the size of a maximal antichain in $2^{n}$, and this can be achieved. We cannot expect to do this well for lattices, but let us show that the number of maximal sublattices can exceed $|L|^{k}$ for any $k$.

Let $S_{n}$ be any set with $n$ elements, $n \in \mathbb{N}$ and $n \geq 5$. We define $\mathbf{L}_{n}$, the Boolean layer cake of degree $n$, to be the lattice - under set inclusion as order relation - of all 2 element and all 3 element subsets of $S_{n}$, together with $\emptyset$ and $S_{n}$ itself. Obviously, $\left|L_{n}\right|=\left(\begin{array}{l}n \\ 2\end{array}\right)+\left(\begin{array}{l}n \\ 3\end{array}\right)+2$, which for large $n$ is approximately $n^{3} / 6$.

THEOREM 1. For any $k \in \mathbb{N}$, there exists an $n$ such that $\mathbf{L}_{n}$ has more than $\left|L_{n}\right|^{k}$ maximal sublattices.

This is proven by counting some rather special maximal sublattices of $\mathbf{L}_{n}$. For any $p \in S_{n}$, let $\mathbf{K}_{p}=\left\{A \subseteq S_{n}: p \notin A\right\} \cup\left\{S_{n}\right\}$. We will describe the maximal sublattices containing $\mathbf{K}_{p}$ for some $p$.

LemMA 2. Let $a, b \in S_{n}, a, b \neq p$. Then $G:=K_{p} \cup\{\{p, a\},\{p, b\}\}$ generates $\mathbf{L}_{n}$. 
PROOF. Consider $x \in S_{n}, x \neq p$. Now $\{p, x\}=(\{p, a\} \cup\{a, x\}) \cap(\{p, b\} \cup\{b, x\})$ and $\{a, x\}$ and $\{b, x\}$ are both in $K_{p}$, so $\{p, x\}$ belongs to the sublattice generated by $G$. It follows that this sublattice contains all 2 element subsets of $S_{n}$ and thus equals $\mathbf{L}_{n}$.

LEMMA 3. Let $a \in S_{n}, a \neq p$. Then $\mathbf{M}_{p, a}:=K_{p} \cup\left\{\{p, a, x\} ; x \in S_{n}\right\}$ is a proper maximal sublattice of $\mathbf{L}_{n}$.

PROOF. It is obvious that $\mathbf{M}_{p, a}$ is a proper sublattice containing the 2 element set $\{p, a\}$. By Lemma 2, adding a further 2 element set (necessarily containing $p$ ) would already generate $\mathbf{L}_{n}$. Adding a further 3 element set means adding some $\{p, x, y\}$ with $\{x, y\} \cap\{p, a\}=\emptyset$. But then $\{p, x\}=\{p, a, x\} \cap\{p, x, y\}$ must also be added, again generating $\mathbf{L}_{n}$ by Lemma 2 .

LEMMA 4. Let $\alpha$ be a maximal collection of pairwise disjoint 2 element subsets of $S_{n}-\{p\}$. Then $\mathbf{M}_{p, \alpha}:=K_{p} \cup\{\{p, x, y\}:\{x, y\} \in \alpha\}$ is a proper maximal sublattice of $\mathbf{L}_{n}$.

PROOF. It is obvious that $\mathbf{M}_{p, \alpha}$ is a proper sublattice. Adding $\{p, a\}$ with $a \neq p$ forces the addition of $\{p, a, x\}=\{p, a\} \cup\{a, x\}$ for any $x \neq p$. Choose $\{x, y\} \in \alpha$ with $a \neq x, y$ (this is possible since $n \geq 5$ ); then we must add $\{p, x\}=\{p, a, x\} \cap$ $\{p, x, y\}$, so all of $\mathbf{L}_{n}$ would be generated by Lemma 2 . If $\{p, u, v\}$ is any 3 element set not in $M_{p . \alpha}$, at least one of $u, v$ - say $u$ - belongs to some $\{x, y\} \in \alpha$. But then adding $\{p, u, v\}$ means adding $\{p, u\}=\{p, u, v\} \cap\{p, x, y\}$, generating again $\mathbf{L}_{n}$ by the first half of this proof.

Although we do not need it to the full extent in proving Theorem 1, we include the following.

COROLlaRY 5. Let $\mathbf{M}$ be any maximal proper sublattice extending $\mathbf{K}_{p}$. Then either $\mathbf{M}=\mathbf{M}_{p, a}$ for some $a \neq p$ or $\mathbf{M}=\mathbf{M}_{p, \alpha}$ for some maximal collection $\alpha$ of pairwise disjoint 2 element subsets of $S_{n}-\{p\}$.

Proof. If $\mathbf{M}$ contains some 2 element set $\{p, a\}$ with $a \neq p, \mathbf{M}$ must be equal to $\mathbf{M}_{p, a}$ by Lemma 3 . So suppose $M-K_{p}$ consists exclusively of 3 element sets $\{p, x, y\}$ and put $\alpha:=\{\{x, y\}:\{p, x, y\} \in M\}$. Any two distinct members of $\alpha$ must be disjoint for otherwise the intersection $\{p, x, y\} \cap\left\{p, x^{\prime}, y^{\prime}\right\}$ would produce a 2 element member $\{p, a\}$ of $M$. Clearly, $\alpha$ will be a maximal collection of such sets and we have $\mathbf{M}=\mathbf{M}_{p, \alpha}$ by Lemma 4 . 
LEMMA 6. For an even integer $k=2 i>0$, let $\tau(k)$ be the number of distinct partitions of a $k$ element set $A$ into 2 element subsets. Then

$$
\tau(k)=\prod_{1 \leq j \leq i}(2 j-1) .
$$

PROOF. Remove an arbitrary point $p$ from $A$ and observe that each partition of $A$ into 2-blocks is uniquely determined by its restriction to $A-\{p\}$. Obviously, there are $(2 i-1) \cdot \tau(2 i-2)$ such traces, which by induction establishes the desired formula.

Assume now that $n \in \mathbb{N}, n=2 i+1 \geq 5$ odd. We count the number of maximal sublattices of type $\mathbf{M}_{p, \alpha}$ of $\mathbf{L}_{n}$. Note that if $p \neq q$ or $\alpha \neq \beta$, then $\mathbf{M}_{p, \alpha} \neq \mathbf{M}_{q, \beta}$. For each $p \in S_{n}$, there are as many sublattices $\mathbf{M}_{p, \alpha}$ as there are partitions of $S_{n}-\{p\}$ into 2 element subsets. Hence their total number in $\mathbf{L}_{n}$ is $n \cdot \tau(n-1)=(2 i+1)$. $\prod_{1 \leq j \leq i}(2 j-1)=\tau(n+1)$. We conclude that $\mathbf{L}_{n}$ has at least $\tau(n+1)$ maximal sublattices. Now

$$
\tau(n+1)=1 \cdot 3 \cdots(2 i+1) \geq 2 \cdot 4 \cdots 2 i=2^{i} i !
$$

while $\left|L_{n}\right|<n^{3} / 6=(2 i+1)^{3} / 6$ for $n \geq 13$, whence

$$
i \geq\left(3\left|L_{n}\right| / 4\right)^{1 / 3}-1 / 2 \geq\left(\left|L_{n}\right| / 2\right)^{1 / 3} .
$$

It follows in particular that, for any $k \in \mathbb{N}, \mathbf{L}_{n}$ has more than $\left|L_{n}\right|^{k}$ maximal sublattices if $n$ is sufficiently large.

This suggests a natural problem.

PROBLEM A. Find an asymptotic upper bound for the number of maximal sublattices in a lattice of size $n$.

Next let us consider the number of maximal sublattices of a modular lattice. Let $\mathbf{L}$ be the lattice of subspaces of a projective plane of order $n=p^{k}$ over a finite field. There are $n^{2}+n+1$ points and $n^{2}+n+1$ lines, so $|L|=2 n^{2}+2 n+4$. Now $\mathbf{L}$ contains maximal sublattices isomorphic to $\mathbf{M}_{n+1 . n+1}$ (the Hall-Dilworth gluing of two copies of $\mathbf{M}_{n+1}$ over an edge); these are obtained by picking a line and a point on it, so there are $\left(n^{2}+n+1\right)(n+1)$ of them. There are also maximal sublattices isomorphic to $\mathbf{M}_{n+1} \times \mathbf{2}$; these are obtained by picking a line and a point not on it, yielding $\left(n^{2}+n+1\right) n^{2}$ of them. For a plane of prime order $p$, these are all the maximal sublattices. So in this case the number of maximal sublattices is roughly $|L|^{2} / 4$.

For a desargean plane of order $p^{2}$, we have in addition maximal sublattices isomorphic to a plane of order $p$. Indeed, any quadrangle (4 points, no 3 collinear) 
generates a sublattice of this type. The number of ordered quadrangles in a plane of order $p^{2}$, divided by the number of ordered quadrangles in a plane of order $p$, is

$$
\frac{\left(p^{4}+p^{2}+1\right)\left(p^{4}+p^{2}\right)\left(p^{4}\right)\left(p^{4}-2 p^{2}+1\right)}{\left(p^{2}+p+1\right)\left(p^{2}+p\right)\left(p^{2}\right)\left(p^{2}-2 p+1\right)} .
$$

Thus $\mathbf{L}$ contains approximately $p^{8}$, or $|L|^{2} / 4$, subplanes of order $p$. Counting those given in the preceding paragraph, a projective plane of order $p^{2}$ has roughly $2 p^{8}$, or $|L|^{2} / 2$, maximal sublattices.

The counting gets harder when you go to fields of order $p^{k}$ with $k>2$, but there are still approximately $n^{4}=p^{8 k}$ maximal sublattices isomorphic to $\mathbf{M}_{n+1, n+1}$ or $\mathbf{M}_{n+1} \times \mathbf{2}$, and roughly $p^{8}$ sublattices isomorphic to a subplane over a maximal subfield, and these are all the maximal sublattices of $\mathbf{L}$. On the other hand, we have been unable to find examples of modular lattices with more than $|L|^{2} / 2$ maximal sublattices. Perhaps that is about as many as you can have.

CONJECTURE B. The number of maximal sublattices of a finite modular lattice is $O\left(|L|^{2}\right)$.

On the other hand, we will show below that finite bounded lattices (and thus in particular distributive lattices) can have at most $|L|$ maximal sublattices. This argument, and other later ones, depend on the following characterization theorem.

THEOREM 7. Let $\mathbf{L}$ be a finite bounded lattice. If $\boldsymbol{u}$ is a canonical meetand of $v$ and $v$ is a canonical joinand of $u$, then $\mathbf{L}-[v, u]$ is a (not necessarily maximal) sublattice of $\mathbf{L}$. Moreover, every maximal sublattice of $\mathbf{L}$ is of this form.

Proof. First suppose $u \in \mathrm{CM}(v)$ and $v \in \mathrm{CJ}(u)$. If $x \vee y \in[v, u]$, then $u=$ $x \vee y \vee \bigvee(C J(u)-\{v\})$. The canonical representation refines this, so $v \leq x \leq u$ or $v \leq y \leq u$. Hence $\mathbf{L}-[v, u]$ is a sublattice. This part requires only semidistributivity.

Now assume that $\mathbf{L}$ is bounded and let $\mathbf{S}$ be a proper sublattice. We will find a pair $u, v$ of the above form with $[v, u] \cap S=\emptyset$.

Let $v_{0}$ be an element of minimal D-rank in $L-S$. Then $v_{0}$ is join irreducible or possibly zero. For if $v_{0}$ were a proper join and in say $\mathrm{D}_{k}(\mathbf{L})$, then its canonical joinands would be in $\mathrm{D}_{k-1}(\mathbf{L})$, and at least one of them would not be in $S$.

There is a canonical meetand $v_{1}$ of $v_{0}$ such that $\left[v_{0}, v_{1}\right] \cap S=\emptyset$. We claim that $\bigvee C \geq v_{0}$ non-trivially implies $\bigvee C \notin v_{1}$. For suppose that $C$ is a non-trivial join cover of $v_{0}$. Since any non-trivial join cover can be refined to a minimal one, there exists a minimal non-trivial join cover $B$ of $v_{0}$ with $v_{0} \leq \bigvee B \leq \bigvee C$. The elements $B$ all have lower D-rank than $v_{0}$, and hence are in $S$. Therefore their join is in $S$, which is disjoint from $\left[v_{0}, v_{1}\right]$. Thus $\bigvee B \notin v_{1}$, and a fortiori $\bigvee C \not v_{1}$. In particular, the 
canonical join representation of $v_{1}$ is not a non-trivial join cover of $v_{0}$, so there is a canonical joinand $v_{2}$ of $v_{1}$ with $v_{0} \leq v_{2} \leq v_{1}$.

For any set $D$ with $v_{1} \geq \wedge D \geq v_{0}$, we have $v_{0}=\wedge D \wedge \wedge\left(\mathrm{CM}\left(v_{0}\right)-\left\{v_{1}\right\}\right)$. The canonical meet representation of $v_{0}$ dually refines this, so $v_{1} \geq d$ for some $d \in D$. In particular, $v_{1} \geq v_{3} \geq v_{2}$ for some $v_{3} \in \mathbf{C M}\left(v_{2}\right)$.

Dually, there exists $v_{4} \in \mathbf{C J}\left(v_{3}\right)$ with $v_{3} \geq v_{4} \geq v_{2}$. Continuing in this way, by finiteness we eventually get $v_{i+2}=v_{i}$, which gives us the desired pair $v_{i}, v_{i+1}$.

It is natural to ask whether this characterization extends to finite semidistributive lattices. We do not know.

Next we want to show that finite bounded lattices do not have too many maximal sublattices. Call a sublattice of the form produced by Theorem 7 special.

THEOREM 8. If $\mathbf{L}$ is a finite bounded lattice, then it has at most $|L|$ special sublattices, and hence at most $|L|$ maximal sublattices.

The proof is by induction on $|L|$. We use the characterization of finite bounded lattices as the lattices obtained from $\mathbf{1}$ by doubling intervals. Clearly the theorem holds for 1.

If $I$ is an interval in the lattice $\mathbf{L}$, let $L[I]$ denote the lattice obtained by doubling $I$.

LEMMA 9. Let $\mathbf{L}$ be a finite lattice with more than 1 element. If $J$ is a join irredundant subset of $\mathbf{L}$ and $K$ is a meet irredundant subset, then $|J|+|K| \leq|L|$.

PROOF. Let $m=\max (|J|,|K|)$. Then $|L| \geq 2^{m} \geq 2 m \geq|J|+|K|$ as desired.

Now let $\mathbf{L}=\mathbf{K}[I]$ where $I=[s, r]$, and assume that $\mathbf{K}$ has at most $|K|$ special sublattices. The join irreducibles of $\mathbf{L}$ are of the form

(1) $p \in \mathbf{J}(\mathbf{K})-I$, or

(2) $(p, 0)$ with $p \in \mathrm{J}(\mathbf{K}) \cap I$, or

(3) $(s, 1)$.

The meet irreducibles of $\mathbf{L}$ are obtained dually.

Moreover, the canonical join representations of elements of $\mathbf{L}$ are obtained from those in $\mathbf{K}$ as follows. For $p \in \mathbf{J}(\mathbf{K})$, let

$$
\widehat{p}= \begin{cases}p & \text { if } p \notin I \\ (p, 0) & \text { if } p \in I .\end{cases}
$$

If $x=t \notin I$ or $x=(t, 0)$, then $\mathrm{CJ}_{\mathrm{L}}(x)=\left\{\widehat{p}: p \in \mathrm{CJ}_{\mathbf{K}}(t)\right\}$. If $x=(t, 1)$, then $\mathrm{CJ}_{\mathbf{L}}(x)=\{(s, 1)\} \cup\left\{\widehat{p}: p \in \mathrm{CJ}_{\mathbf{K}}(t)\right.$ and $\left.p \nless s\right\}$. The canonical meet representations in $\mathbf{L}$ are of course given dually. 
We can describe the special sublattices of $\mathbf{L}$ as follows. Let $\mathbf{S}=\mathbf{L}-[v, u]$ with $u \in \mathrm{CM}(v)$ and $v \in \mathrm{CJ}(u)$.

(1) If $v \in \mathbf{J}(\mathbf{K})-I$ and $u \in \mathbf{M}(\mathbf{K})-I$, then $\mathbf{K}-[v, u]$ is a special sublattice of $\mathbf{K}$.

(2) If $v \in \mathrm{J}(\mathbf{K})-I$ and $u=(q, 1)$ with $q \in \mathbf{M}(\mathbf{K}) \cap I$, then $v \not \leq s$ and $\mathbf{K}-[v, q]$ is a special sublattice of $\mathbf{K}$. (If $v<s$ and $q \in I$, then $\mathbf{K}-[v, q]$ may be special, but $\mathbf{L}-[v,(q, 1)]$ will not be, as $(s, 1)$ is a canonical joinand of $(q, 1)$. So you might lose some special sublattices.)

(3) If $v \in \mathbf{J}(\mathbf{K})-I$, then $u \neq(r, 0)$ since the latter is not a canonical meetand of $u$.

(4) The case $v=(p, 0)$ with $p \in \mathrm{J}(\mathbf{K}) \cap I$ and $u \in \mathbf{M}(\mathbf{K})-I$ is the dual of (2), and so yields no new sublattices.

(5) If $v=(p, 0)$ with $p \in \mathrm{J}(\mathbf{K}) \cap I$ and $u=(q, 1)$ with $q \in \mathbf{M}(\mathbf{K}) \cap I$, then $\mathbf{K}-[p, q]$ is a special sublattice of $\mathbf{K}$. (If $p=s$ or $q=r$, then $\mathbf{K}-[p, q]$ may be special, but $\mathbf{L}-[(p, 0),(q, 1)]$ will not be, as in (2).)

(6) If $v=(p, 0)$ with $p \in \mathrm{J}(\mathbf{K}) \cap I$ and $u=(r, 0)$, then $p$ is a canonical joinand of $r$ in $\mathbf{K}$. Hence the number of special sublattices of this type is $|\mathrm{CJ}(r) \cap I|$, which is a join irredundant set.

(7) The case $v=(s, 1)$ and $u \in \mathrm{M}(\mathbf{K})-I$ is the dual of (3), and does not occur.

(8) The case $v=(s, 1)$ and $u=(q, 1)$ with $q \in \mathbf{M}(\mathbf{K}) \cap I$ is the dual of $(6)$, and yields $|\mathrm{CM}(s) \cap I|$ new sublattices.

(9) If $v=(s, 1)$, then $u \neq(r, 0)$ because $(s, 1) \not \leq(r, 0)$.

Thus the number of special sublattices in $\mathbf{L}$ is at most the number of those in $\mathbf{K}$ plus $|\mathrm{CJ}(r) \cap I|+|\mathrm{CM}(s) \cap I|$. On the other hand, $|L|=|K|+|I|$. So unless $I$ is a single point, we are done by the lemma applied to $I$.

If $I$ consists of a doubly reducible point $p$, then cases (6) and (8) do not occur, and we get no new special sublattices. If $p$ is join irreducible only, then (6) yields one new special sublattice and (8) does not occur. If $p$ is meet irreducible only, then dually (8) yields exactly one new special sublattice. If $p$ is doubly irreducible, (6) and (8) give two special sublattices where there was one before. So in every case we get at most one more, and this completes the proof of the theorem.

Note that we also have the estimate that, for bounded lattices, the number of maximal sublattices is at most $|\mathbf{J}(\mathbf{L})||\mathbf{M}(\mathbf{L})|$, which can be either better or worse than the bound $|L|$ given by Theorem 8 .

\section{The size of maximal sublattices}

If $\mathbf{S}$ is a maximal sublattice of $\mathbf{L}$, we know that $|S|$ can be as large as $|L|-1$.

At the other extreme, Figure 1 shows an infinite lattice which has a maximal sublattice isomorphic to the five element modular, non-distributive lattice $\mathbf{M}_{3}$ (indicated by solid points). An obvious modification shows that $\mathbf{M}_{3}$ is also a maximal sublattice 
of arbitrarily large finite lattices. Easy arguments show that no four element lattice has these properties.

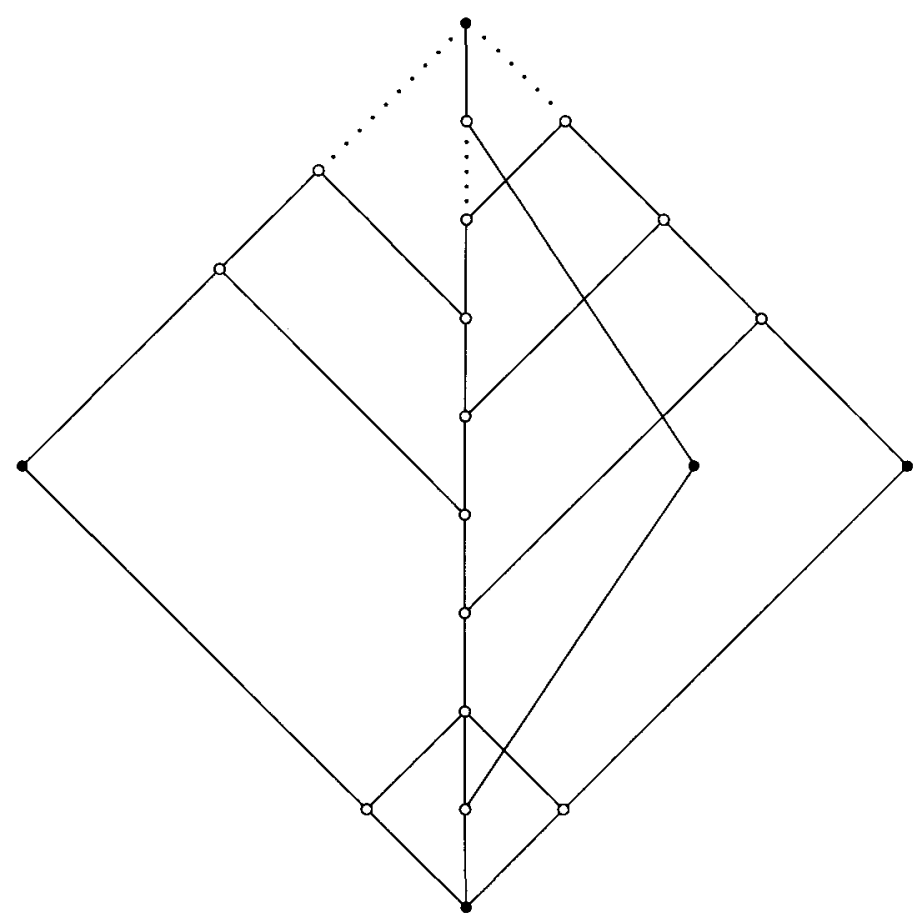

FIGURE 1

This leads to an interesting question.

PROBLEM C. Which finite lattices are isomorphic to a maximal sublattice of an infinite lattice? of arbitrarily large finite lattices? Are these properties the same?

This problem will be addressed in [8]. For example, it is shown there that if the five element nonmodular lattice $\mathbf{N}_{5}$ is isomorphic to a maximal sublattice of a lattice L, then $|L| \leq 8$.

However, note that if $\mathscr{V}$ is a locally finite variety, $\mathbf{L} \in \mathscr{V}$ and $\mathbf{S}$ is a maximal sublattice of $\mathbf{L}$, then $|L| \leq\left|\mathscr{F}_{\mathscr{V}}(|S|+1)\right|$. Thus, for each $k$, there is an unbounded, non-decreasing function $\sigma_{k}(n)$ such that if $\mathbf{L}$ is a finite lattice in $\mathscr{B}_{k}$ (the variety generated by the finite bounded lattices with $\mathrm{J}(\mathbf{L}) \subseteq \mathrm{D}_{n}(\mathbf{L})$ ) and $\mathbf{S}$ is a maximal sublattice of $\mathbf{L}$, then $|S| \geq \sigma_{k}(|L|)$.

PROBLEM D. Find an asymptotic lower estimate for $\sigma_{k}(n)$. 
For example, Rival proved in [13] that if $\mathbf{S}$ is a maximal sublattice of a distributive lattice $\mathbf{L}$ with $|L| \geq 3$, then $|S| \geq|L| / 3$. This can be derived from Lemma 3 and the fact that every special sublattice of a finite distributive lattice is maximal. Thus $\sigma_{0}(n) \geq 2 n / 3$. On the other hand, for any lattice $\mathbf{D}$, the direct product $\mathbf{3} \times \mathbf{D}$ has a maximal sublattice

$$
\mathbf{S}=\left\{\left\langle x_{0}, x_{1}\right\rangle \in\{0,1,2\} \times \mathbf{D}: x_{0} \neq 1\right\}
$$

with $2|D|$ elements. Hence $\sigma_{0}(3 k)=2 k$, and more generally $\sigma_{0}(n)=\lceil 2 n / 3\rceil$.

Let us show that this situation is rather special to distributive lattices: there exist finite bounded lattices with maximal sublattices such that $|S| /|L|$ is arbitrarily small.

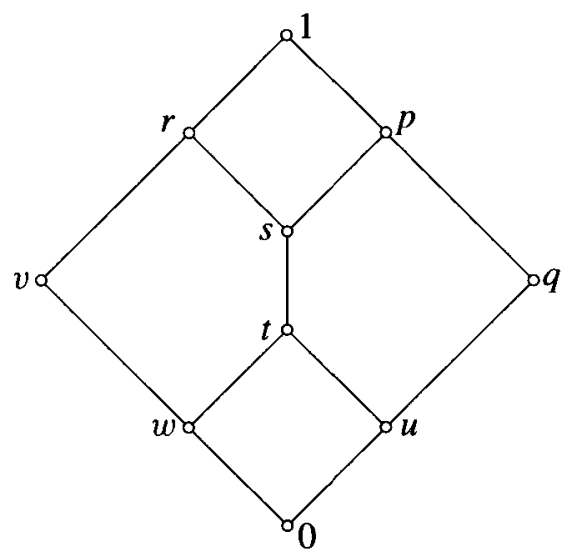

FIGURE 2

THEOREM 10. Let $\mathbf{K}$ be the lattice in Figure 2. For every $\varepsilon>0$ there exists a finite lattice $\mathbf{L} \in \mathscr{V}(\mathbf{K})$ with a maximal sublattice $\mathbf{S}$ such that $|S| /|L|<\varepsilon$.

Note that as $\mathbf{K} \in \mathscr{B}_{1}$, every lattice in the variety $\mathscr{V}(\mathbf{K})$ is also in $\mathscr{B}_{1}$. Hence $\lim _{n \rightarrow \infty} \sigma_{1}(n) / n=0$.

PROOF. There is a homomorphism of $\mathbf{K}$ onto the 3 element chain whose kernel $\kappa$ has 3 blocks:

$$
B_{1}=\{1, p, q\}, \quad B_{r}=\{r, s, t, u\}, \quad B_{0}=\{v, w, 0\} .
$$

Let $\mathbf{L}_{n}$ be the subalgebra $B_{0}^{n} \cup B_{r}^{n} \cup B_{1}^{n}$ of the direct power $\mathbf{K}^{n}$, that is,

$$
L_{n}=\left\{x \in K^{n}: x_{i} \kappa x_{j} \text { for all } i, j\right\}
$$


We claim that $\mathbf{S}_{n}=B_{0}^{n} \cup B_{1}^{n}$ is a maximal sublattice of $\mathbf{L}_{n}$. As $\left|S_{n}\right|=2 \cdot 3^{n}$ and $\left|L_{n}\right|=2 \cdot 3^{n}+4^{n}$, this will prove the theorem.

Let $x$ be any element of $B_{r}^{n}$, and let $\bar{v}$ be the element with $\bar{v}_{i}=v$ for all $i$; similarly $\bar{r}=\langle r, \ldots, r\rangle$. Then $\bar{v} \in S_{n}$ and $\bar{v} \vee x=\bar{r}$, so that $\bar{r}$ is in the sublattice generated by $S_{n} \cup\{x\}$. Hence it suffices to prove that $S_{n} \cup\{\bar{r}\}$ generates $\mathbf{L}_{n}$, that is, $B_{r}^{n}$ is contained in the sublattice generated by $S_{n} \cup\{\bar{r}\}$.

Again let $x$ be any element of $B_{r}^{n}$, and let

$$
\begin{array}{ll}
R=\left\{i: x_{i}=r\right\}, & S=\left\{i: x_{i}=s\right\} \\
T=\left\{i: x_{i}=t\right\}, & U=\left\{i: x_{i}=u\right\} .
\end{array}
$$

Define elements $y \in B_{1}^{n}$ and $z \in B_{0}^{n}$ by

$$
y_{i}=\left\{\begin{array}{ll}
1 & \text { if } i \in R \\
p & \text { if } i \in S \\
q & \text { if } i \in T \cup U
\end{array} \quad z_{j}= \begin{cases}v & \text { if } i \in R \\
w & \text { if } i \in S \cup T \\
0 & \text { if } i \in U .\end{cases}\right.
$$

Then $x=(\bar{r} \wedge y) \vee z$, which is in the sublattice generated by $S_{n} \cup\{\bar{r}\}$. By the preceding reduction, this shows that $\mathbf{S}_{n}$ is a maximal sublattice of $\mathbf{L}_{n}$.

\section{Frattini sublattices and varieties}

If $\mathbf{C}$ is a chain, then $|\Phi(\mathbf{C})|=0$. Otherwise, so long as $|L|>4$, we have

$$
2 \leq|\Phi(\mathbf{L})| \leq|L|-3
$$

with the lower limit being obtained by $\mathbf{M}_{n}$ 's and the upper limit by relatively free lattices, so there does not seem to be much more to say there.

We want to consider the third problem from the introduction: For which lattice varieties $\mathscr{V}$ is it true that every finite non-trivial lattice $\mathbf{L} \in \mathscr{V}$ is isomorphic to $\Phi\left(\mathbf{L}^{\prime}\right)$ for some finite lattice $\mathbf{L}^{\prime} \in \mathscr{V}$ ? It is useful to let $\Phi\left(\mathscr{V}_{f}\right)$ denote the set of all lattices isomorphic to some $\Phi(\mathbf{K})$ where $\mathbf{K}$ is a finite lattice in $\mathscr{V}$.

The known results on this problem can be summarized as follows. If $\mathbf{L}$ is a finite lattice and not a chain, then $\Phi(\mathbf{L})$ must contain the largest join reducible and smallest meet reducible elements. Thus $|\Phi(\mathbf{L})| \neq 1$ when $\mathbf{L}$ is finite. Koh showed that every finite lattice with $|L|>1$ is in $\Phi\left(\mathscr{L}_{f}\right)$ where $\mathscr{L}$ is the variety of all lattices [10]. Moreover, Adams and Sichler proved that every lattice $\mathbf{L}$ is isomorphic to $\Phi(\mathbf{K})$ for some $\mathbf{K} \in \mathscr{V}(\mathbf{L})$ with $|K|=|L|+\aleph_{0}$, see [3]. However, Adams in [2], and independently Chen, Koh and Tan in [4], showed that for distributive lattices $2 \times 2 \notin \Phi\left(\mathscr{D}_{f}\right)$. Eventually, Abad and Adams characterized the lattices in $\Phi\left(\mathscr{D}_{f}\right)$, though the characterization is necessarily a bit complicated; see [1]. 
THEOREM 11. There exist infinitely many lattice varieties $\mathscr{C}$ such that every finite non-trivial lattice $\mathbf{L} \in \mathscr{C}$ is isomorphic to $\Phi\left(\mathbf{L}^{\prime}\right)$ for some finite lattice $\mathbf{L}^{\prime} \in \mathscr{C}$, that is, $\mathscr{C}_{f} \subseteq \Phi\left(\mathscr{C}_{f}\right)$.

PrOOF. Koh's construction gives, for each finite non-trivial lattice $\mathbf{L}$, a finite lattice $\mathbf{L}^{\prime}$ such that $\Phi\left(\mathbf{L}^{\prime}\right) \cong \mathbf{L}$. This he does by replacing every covering relation of $\mathbf{L}$ by $2 \times 2$. In fact, there are infinitely many varieties which are closed under Koh's construction.

Let $\mathbf{K}$ be a primitive lattice, that is, a finite, subdirectly irreducible, projective lattice. (Equivalently, $\mathbf{K}$ is finite, subdirectly irreducible, bounded, and satisfies Whitman's condition (W).) Then the class of all lattices not containing a sublattice isomorphic to $\mathbf{K}$ is a variety $\mathscr{C}_{\mathbf{K}}$. If further $\mathbf{K}$ contains no doubly irreducible elements, then it is clear that $\mathscr{C}_{\mathrm{K}}$ is closed under Koh's construction. Thus for each finite non-trivial lattice $\mathbf{L} \in \mathscr{C}_{\mathbf{K}}$, there exists a finite lattice $\mathbf{L}^{\prime} \in \mathscr{C}_{\mathbf{K}}$ with $\Phi\left(\mathbf{L}^{\prime}\right) \cong \mathbf{L}$.

Ježek and Slavík give three infinite lists of primitive lattices with no doubly irreducible elements in [9].

Now recall that not every finite distributive lattice can be represented as $\Phi(\mathbf{K})$ with $\mathbf{K} \in \mathscr{D}_{f}\left(=\mathscr{B}_{0 f}\right)$. In fact, this is the only variety for which we know that $\mathscr{V}_{f} \nsubseteq \Phi\left(\mathscr{V}_{f}\right)$. Repeated failed attempts to represent certain lattices have given us some faith in the following conjecture.

CONJECTURE E. For every $n \geq 0$ there is a finite non-trivial lattice $\mathbf{L} \in \mathscr{B}_{n}$ which cannot be represented as $\Phi(\mathbf{K})$ with $\mathbf{K}$ a finite lattice in $\mathscr{B}_{n}$, that is, $\mathscr{B}_{n f} \nsubseteq \Phi\left(\mathscr{B}_{n f}\right)$.

However, let us show that $\mathscr{B}_{n} \subseteq \Phi\left(\mathscr{B}_{n+1 f}\right)$.

THEOREM 12. Every finite non-trivial lattice $\mathbf{L} \in \mathscr{B}_{n}$ can be represented as $\Phi(\mathbf{K})$ for some finite lattice $\mathbf{K} \in \mathscr{B}_{n+1}$.

PROOF. Let $\mathbf{P}$ be the ordered set of (nonzero) join irreducible elements of the finite lattice $\mathbf{L} \in \mathscr{B}_{n}$. We may assume that $|\mathbf{L}|>2$, as we can represent $\mathbf{2}$ as $\Phi(\mathbf{2} \times \mathbf{2})$. We may further assume that $L$ is linearly indecomposable, for we can use either $2 \times 2$ or the construction below for each linear summand of $L$. This means that $P$ cannot be written as the disjoint union of two nonempty sets, $P=A \dot{U} B$, with $a \geq b$ whenever $a \in A$ and $b \in B$.

It is useful before proceeding to identify a particular type of element in $\mathbf{L}$. Let us say that $p$ is a splitting element for $\mathbf{P}$ if there exists a unique element $r \in P$ minimal with respect to the property $r \not \leq p$. Every element of $P$ is then either below $p$ or above $r$, so that $p$ is the unique element which is maximal with respect to $p \nsupseteq r$. In this case, we will say that $\langle p, r\rangle$ is a splitting pair for $\mathbf{P}$, and write $p=\mu(r)$ and $r=v(p)$. According to this terminology, $v(p)$ need not be a splitting element. 
An element $p$ is doubly irreducible if it is in $P$ (that is, join irreducible) and has a unique upper cover $p^{*}$. It is easy to see that splitting elements are doubly irreducible in $\mathbf{L}$, but unless $\mathbf{L}$ is distributive the converse need not hold.

We construct $\mathbf{K}$ as follows. Let $\mathbf{Q}$ be the ordered set consisting of all $(p, 0)$, $(p, 1,0)$ and $(p, 1,1)$ for $p \in P$, and $(p, 1,2)$ if $p$ is a splitting element in $\mathbf{P}$. Order $\mathbf{Q}$ lexicographically. Then $\mathbf{K}$ is isomorphic to the join semilattice with zero generated by $\mathbf{Q}$ subject to the relations

(I) $(p, 1,0) \leq \bigvee_{q \in U}(q, 1,0)$ if $p \leq \bigvee U$ in $\mathbf{L}$,

(II) $(p, 1,1) \leq(p, 1,0) \vee(q, 0)$ if $q \notin p$,

(III) $(p, 1,2) \leq(p, 1,0) \vee(\nu(p), 1,0)$ if $p$ is a splitting element.

This construction is partially illustrated in Figure 3. Since $\mathbf{K}$ is a finite join semilattice with zero, it is a lattice.
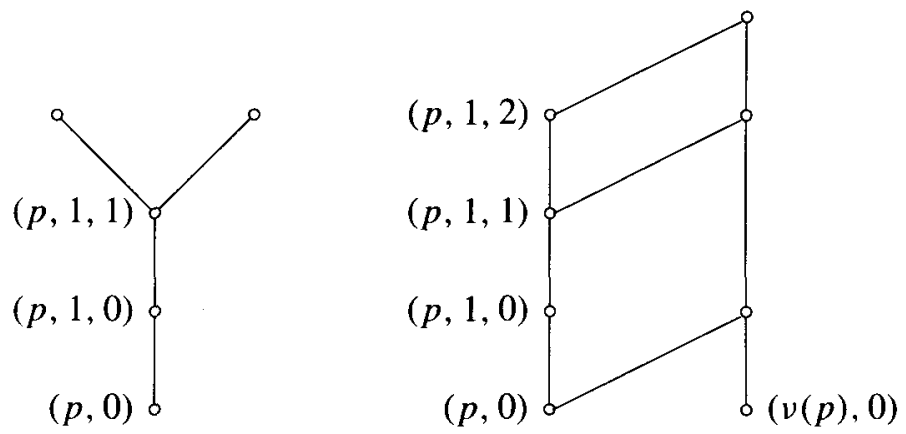

FIGURE 3

Before proceeding, we need to work out some of the arithmetic of $\mathbf{K}$ (in terms of that of $\mathbf{L}$ ). Let us say that a set $X \subseteq Q$ is closed if, for all $q \in Q, q \leq \vee X$ implies $q \in X$. Clearly, $X$ is closed if and only if there exists $x \in K$ such that $X=\{q \in Q: q \leq x\}$. We want to determine the closed subsets of $Q$.

As a matter of notation, for $p \in P$ let $m_{p}=2$ if $p$ is a splitting element, and $m_{p}=1$ otherwise. Also, for $q \in Q$ let $\bar{q}$ denote $\{u \in Q: u \leq q\}$.

LEMMA 13. A subset $X \subseteq Q$ is closed if and only if it is of one of the following forms.

(1) $\overline{(p, 0)}$ or $\overline{(p, 1,0)}$ or $\overline{(p, 1,1)}$ or (for splitting elements) $\overline{(p, 1,2)}$.

(2) $\overline{(p, 1,1)} \cup\{(v(p), 0)\}$ where $p$ is a splitting element of $\mathbf{P}$.

(3) For some $a \in L$ and $R \subseteq P, \bigcup_{p \leq a} \overline{\left(p, 1, m_{p}\right)} \cup\{(r, 0): r \in R\}$ where $r \not \leq a$ and $r_{*} \leq$ a for all $r \in R$. 
Moreover, if $X$ is closed and $x=\bigvee X$ in $\mathbf{K}$, then $x$ has one of the following canonical join representations in $\mathbf{K}$.

(4) $(p, 0)$ or $(p, 1,0)$ or $(p, 1,1)$ or (for splitting elements) $(p, 1,2)$.

(5) $(p, 1,0) \vee(v(p), 0)$ where $p$ is a splitting element of $\mathbf{P}$.

(6) $\bigvee\{(p, 1,0): p \in \mathrm{CJ}(a)$ and $p \not \leq r$ for all $r \in R\} \vee \bigvee\{(r, 0): r \in R\}$.

In particular, since every element of $\mathbf{K}$ has a canonical join representation, $\mathbf{K}$ satisfies $\left(\mathrm{SD}_{\vee}\right)$.

It is not hard to see that the sets given in the Lemma 13 are closed. Conversely, assume that $X$ is closed, and let $a=\bigvee\{p \in P:(p, 1,0) \in X\}$. (Possibly $a=0$.) The case $X=\emptyset$ is contained in (3), and the case when $X$ has a unique maximal element is (1). If $X$ has at least two maximal elements, then $(p, 1,0) \in X$ implies $\left(p, 1, m_{p}\right) \in X$, with the exception given in case (2).

Let $R=\{r \in P:(r, 0) \in X$ but $(r, 1,0) \notin X\}$. If $r \in R$, then by closure rule (I) $r \not \leq a$, but for all $p<r$ we have $(p, 1,0)<(r, 0)$, whence $(p, 1,0) \in X$ and $p \leq a$; thus $r_{*} \leq a$. Thus every closed set has the form (1), (2) or (3).

Let $C$ denote the set of joinands given in (4), (5) or (6). In each case we have $C \subseteq X$ and $q \in X$ implies $q \leq \bigvee C$, whence $\bigvee C=\bigvee X=x$. To see that these joins are canonical, we need to show that if $U \subseteq Q$ and $x=\bigvee U$, then each element of $C$ is below some element of $U$. (Certainly it suffices to consider the case when the elements of $U$ are join irreducible.)

The elements in case (1) are join irreducible, so they are their own canonical joinands (4). For case (2), $X-\{(v(p), 0)\}=\overline{(p, 1,1)}$ is closed, so $(v(p), 0)$ is needed for the join. On the other hand,

$$
\begin{aligned}
(p, 0) \vee(v(p), 0) & \left.=\bigcup_{q \leq p_{*}} \overline{\left(q, 1, m_{q}\right)} \cup\{(p, 0)\} \cup\{v(p), 0)\right\} \\
& =X-\{(p, 1,0),(p, 1,1)\} .
\end{aligned}
$$

As $U$ is not contained in this set, but is contained in $X$, it must contain one of $(p, 1,0)$ or $(p, 1,1)$.

In case (3), it is clear that $X-\{(r, 0)\}$ is closed for each $r \in R$. As $U \subseteq X$ and $U \nsubseteq X-\{(r, 0)\}$ for every $r \in R$, we have $\{(r, 0): r \in R\} \subseteq U$. So suppose $p \in \mathrm{CJ}(a)$ and that $p \not \leq r$ for all $r \in R$. Now $\kappa(p)$ exists in $\mathbf{L}$. We claim that

$$
Y=\bigcup_{q \leq \kappa(p)} \overline{\left(q, 1, m_{q}\right)} \cup\{(p, 0)\} \cup\{(r, 0): r \in R\}
$$

is a closed set which does not contain $(p, 1,0)$. Of course, $p_{*} \leq \kappa(p)$. If $r \in R$, then $p \neq r$ as $p \leq a$ and $r \not \leq a$. However, $r_{*} \leq a$, and every element below $a$ is either above $p$ or below $\kappa(p)$, since $p$ is a canonical joinand of $a$. As $p \not \leq r *$, we have $r_{*} \leq \kappa(p)$. Thus $Y$ is indeed closed. 
Now $U \nsubseteq Y$, so choose $u \in U-Y$. Then $u$ is either of the form $\left(s, 1, k_{s}\right)$ or $(s, 0)$. In either case $s \leq a$ and $s \not \leq \kappa(p)$, so $p \leq s$. Also $u \neq(p, 0)$. Thus either $u=\left(s, 1, k_{s}\right)$ with $s \geq p$, or $u=(s, 0)$ with $s>p$. Regardless, we have $u \geq(p, 1,0)$, as desired. This proves Lemma 13 .

Now let us verify that $Q \subseteq \mathrm{D}_{n+1}(\mathbf{K})$. It follows from the above proof that if $U$ is a minimal non-trivial join cover of an element $q \in Q$, then every element of $U$ is of the form $(s, 1,0)$. For certainly a minimal join cover of $q$ consists of the canonical joinands of $\bigvee U$. And if $q \in \bigcup_{p \leq a} \overline{\left(p, 1, m_{p}\right)} \cup\{(r, 0): r \in R\}$ as above, and $q \neq(r, 0)$ for all $r \in R$, then $q \in \bigcup_{p \leq a} \overline{\left(p, 1, m_{p}\right)}$, so for a minimal non-trivial join cover we must have $R$ empty.

An easy induction now shows that if $p \in \mathrm{D}_{k}(\mathbf{L})$, then $(p, 0)$ and $(p, 1,0)$ are in $\mathrm{D}_{k}(\mathbf{K})$. (Use the fact that $(p, 1,0) \leq \bigvee_{s \in S}(s, 1,0)$ in $\mathbf{K}$ if and only if $p \leq \bigvee S$ in L.) Since $P \subseteq \mathrm{D}_{n}(\mathbf{L})$, all the elements $(p, 1,1)$ and (if $p$ is splitting) $(p, 1,2)$ are in $\mathrm{D}_{n+1}(\mathbf{K})$, because all their minimal non-trivial join covers are contained in $\mathrm{D}_{n}(\mathbf{K})$.

To check that $\mathbf{K}$ is indeed in $\mathscr{B}_{n+1}$, it remains to prove that $\mathbf{K}$ satisfies $\left(\mathrm{SD}_{\wedge}\right)$. For this, it suffices to show that $\kappa(q)$ exists in $\mathbf{K}$ for each $q \in Q$. Since the elements $\kappa(q)$ are precisely the meet irreducible elements of $\mathbf{K}$, and we eventually want to find all the special sublattices of $\mathbf{K}$, we will also want to apply the Lemma 13 to find the canonical join expressions of these elements.

A couple of cases are easy: $\kappa((p, 1,1))=(p, 1,0)$ since $(p, 1,0)$ is doubly irreducible, and if $p$ is a splitting element,

$$
\kappa((p, 1,2))=(p, 1,1) \vee(\nu(p), 0)=(p, 1,0) \vee(\nu(p), 0)
$$

with the latter expression being canonical. Moreover, if $p$ is a splitting element, $\kappa((\nu(p), 0))=(p, 1,2)$. Thus it remains to find $\kappa((p, 0))$ with $p \neq \nu(q)$, and $\kappa((p, 1,0))$. The above cases cover the situations where $\kappa(q)$ will have the form (1) or (2) of the Lemma 13. (Recall that $\kappa$ is a one-to-one correspondence between join and meet irreducibles in a finite semidistributive lattice.)

To find $\kappa((p, 0))$, we note that

$$
U=\bigcup_{q \leq \kappa(p)} \overline{\left(q, 1, m_{q}\right)} \cup\left\{(r, 0): r \not \leq \kappa(p) \text { and } r_{*} \leq \kappa(p) \text { and } r \neq p\right\}
$$

is a closed set which does not contain $(p, 0)$, but does contain all $w \in Q$ properly below $(p, 0)$. Furthermore, if $v \in Q-U$, then either $v=(p, 0)$ or $v \geq(s, 1,0)$ for some $s \not \leq \kappa(p)$. In the latter case we have $p \leq p_{*} \vee s$, that is, there exist $q_{1}, \ldots, q_{k} \leq p_{*}$ such that $p \leq s \vee \bigvee q_{j}$. Then $\left(q_{j}, 1,0\right)<(p, 0)$ for all $j$, and

$$
(p, 1,0) \leq(s, 1,0) \vee \bigvee_{1 \leq j \leq k}\left(q_{j}, 1,0\right) \leq v \vee(p, 0)_{*}
$$

so that $U$ is the largest closed set containing $\{w \in Q: w<(p, 0)\}$ but not containing $(p, 0)$. Thus $\bigvee U=\kappa((p, 0))$. By the Lemma 13, if we let

$$
R_{p}=\left\{\left(r \in P: r \not \leq \kappa(p) \text { and } r_{*} \leq \kappa(p) \text { and } r \neq p\right\}\right.
$$


then we have canonically

$\kappa((p, 0))$

$=\bigvee\left\{(q, 1,0): q \in \mathrm{CJ}(\kappa(p))\right.$ and $q \not \leq r$ for all $\left.r \in R_{p}\right\} \vee \bigvee\left\{(r, 0): r \in R_{p}\right\}$.

The same argument shows that if we take $V=U \cup\{(p, 0)\}$, then $\bigvee V=$ $\kappa((p, 1,0))$. Thus, with $S_{p}=R_{p} \cup\{p\}$, we have the canonical join expression

$$
\begin{aligned}
& \kappa((p, 1,0)) \\
& \quad=\bigvee\left\{(q, 1,0): q \in \mathrm{CJ}(\kappa(p)) \text { and } q \not \leq r \text { for all } r \in S_{p}\right\} \vee \bigvee\left\{(r, 0): r \in S_{p}\right\} .
\end{aligned}
$$

Thus $\kappa(q)$ exists for all $q \in Q$, and $\mathbf{K}$ satisfies $\left(\mathrm{SD}_{\wedge}\right)$.

Recall that we are trying to find the pairs $u, v \in K$ with $u \in \mathrm{CM}(v)$ and $v \in \mathrm{CJ}(u)$. In order to do this, we must write each join irreducible as a canonical meet of meet irreducible elements. However, since the elements $(p, 1,1)$ are not canonical joinands of any meet irreducible $\kappa(q)$, we need not do those.

The elements of the form $(p, 1,0)$ or $(p, 1,2)$ are doubly irreducible.

This leaves the elements of the form $(p, 0)$. Since $(p, 1,0)$ covers $(p, 0)$, we have $(p, 0)=(p, 1,0) \wedge \kappa((p, 1,0))$, so the canonical meetands of $(p, 0)$ are $\kappa((p, 1,0))$ and some set $T$ of meet irreducible elements above $(p, 1,0)$. Now $(p, 0)$ is a canonical joinand of $\kappa((p, 1,0))$. We claim that $(p, 0)$ is not a canonical joinand of any member of $T$. So let $t \in T$. If $p=v(q)$ for some splitting element $q$, then $\kappa((q, 1,2))=(q, 1,0) \vee(p, 0)$, but that is below $\kappa((p, 1,0))=(q, 1,2) \vee(p, 0)$, and thus is not in $T$. So we may assume that the canonical form of $t$ is given by (6) of the Lemma 13. As $(p, 1,0) \leq t$, we have $p \leq a$, and hence $(p, 0)$ is not a canonical joinand of $t$.

Thus the special sublattices of $\mathbf{K}$ consist of the complements of the doubly irreducible elements of the form $(p, 1,0)$ or $(p, 1,2)$, and the complements of the intervals $[(p, 0), \kappa((p, 1,0))]$. We must check that none of these intervals contains a doubly irreducible or another such interval, so that the special sublattices are indeed maximal. If

$$
(p, 0) \leq(q, 1, j) \leq \kappa((p, 1,0))=\bigvee_{t \leq \kappa(p)}\left(t, 1, m_{t}\right) \vee \bigvee_{\substack{r_{*} \leq \kappa(p) \\ r \notin \alpha \kappa(p)}}(r, 0)
$$

then $p \leq q \leq \kappa(p)$, which is impossible. Thus the intervals contain no doubly irreducible element. Now suppose

$$
(p, 0) \leq(q, 0) \leq \kappa((q, 1,0)) \leq \kappa((p, 1,0)) .
$$

Note that $\kappa((q, 1,0)) \leq \kappa((p, 1,0))$ implies $\kappa(q) \leq \kappa(p)$, for if $s \leq \kappa(q)$ then $(s, 1,1) \leq \kappa((q, 1,0))$, whence $(s, 1,1) \leq \kappa((p, 1,0))$ and $s \leq \kappa(p)$. Thus the above inclusions imply $p \leq q$ and $\kappa(q) \leq \kappa(p)$. But if $p$ and $q$ are distinct, then 
$\kappa(q) \neq \kappa(p)$, and so $q \leq \kappa(q)^{*} \leq \kappa(p)$. Combining these inequalities yields $p \leq q \leq \kappa(p)$, a contradiction. Thus the above inclusions hold only when $p=q$.

The interval $[(p, 0), \kappa((p, 1,0))]$ consists of precisely the elements of $\mathbf{K}$ which have $(p, 0)$ as a canonical joinand. To see this, first suppose that $(p, 0)$ is a canonical joinand of $x$. Then, by the Lemma 13, either $p=v(q)$ for some splitting element $q$ and $x=(q, 1,0) \vee(p, 0) \leq \kappa((p, 1,0))$, or $x$ has the form given in (6) with $p \in R$. In the latter case, $p_{*} \leq a$ and $p \leq \leq a$, so $a \leq \kappa(p)$. For any canonical joinand $u$ of $x$, either $u=(s, 1,0)$ with $s \leq a \leq \kappa(p)$, or $u=(r, 0)$ with $r \not \leq a$ and $r_{*} \leq a$. In the latter case, either $r \leq \kappa(p)$, and so $(r, 1,0) \leq \kappa((p, 1,0))$, or $(r, 0)$ is a canonical joinand of $\kappa((p, 1,0))$. Thus every canonical joinand of $x$, and hence $x$ itself, is below $\kappa((p, 1,0))$. On the other hand, using the Lemma 13 and the observation above that $[(p, 0), \kappa((p, 1,0))]$ contains no join irreducibles besides $(p, 0)$, it is easy to see that if $x \in[(p, 0), \kappa((p, 1,0))]$, then $(p, 0)$ is a canonical joinand of $x$ (either by $(6)$ or (5)).

We conclude that $\Phi(\mathbf{K})$ consists of those elements $x \in K$ such that for all $p \in P$, $x \neq(p, 1,0), x \neq(p, 1,2)$, and $(p, 0)$ is not a canonical joinand of $x$. This last condition means that if $(p, 0) \leq x$ then $(p, 1,0) \leq x$; since $x \neq(p, 1,0)$, either $x=(p, 1,1)$ or $(q, 0) \leq x$ for some $q \not \leq p$, and hence $(p, 1,1) \leq x$. Moreover, if $(p, 1,2) \leq x$, then $x \neq(p, 1,2)$ so $(\nu(p), 0) \leq x$, whence $(v(p), 1,0) \leq x$; thus $x$ can be expressed as a join of elements not involving $(p, 1,2)$ by the relations.

Thus $\Phi(\mathbf{K})$ is the join subsemilattice (with zero) of $\mathbf{K}$ generated by the elements $(p, 1,1)$ with $p \in P$. It remains only to show that this is order isomorphic to $\mathbf{L}$, since $\Phi(\mathbf{K})$ is a sublattice of $\mathbf{K}$. This is a straightforward consequence of our previous calculations.

Thus if we could prove Conjecture E we would have that $\mathscr{B}_{n f} \nsubseteq \Phi\left(\mathscr{B}_{n f}\right)$, but $\mathscr{B}_{n f} \subseteq \Phi\left(\mathscr{B}_{n+1 . f}\right)$. This would require some work and insight. Perhaps something similar is true for $n$-distributive modular lattices. Indeed, it would be good to find any more examples where $\mathscr{V}_{f} \nsubseteq \Phi\left(\mathscr{V}_{f}\right)$.

\section{References}

[1] Manuel Abad and M. E. Adams, 'The Frattini sublattice of a finite distributive lattice', Algebra Universalis 32 (1994), 314-329.

[2] M. E. Adams, 'The Frattini sublattice of a distributive lattice', Algebra Universalis 3 (1973), 216-228.

[3] M. E. Adams and J. Sichler, 'Frattini sublattices in varieties of lattices', Colloq. Math. 44 (1982), 181-184.

[4] C. C. Chen, K. M. Koh and S: K. Tan, 'On the Frattini sublattice of a finite distributive lattice', Algebra Universalis 5 (1975), 88-97. 
[5] A. Day, 'Splitting lattices generate all lattices', Algebra Universalis 7 (1977), 163-170.

[6] - 'Characterizations of finite lattices that are bounded-homomorphic images or sublattices of free lattices', Canad. J. Math. 31 (1979), 69-78.

[7] Freese, J. Ježek and J. B. Nation, Free Lattices, volume 42 of Mathematical Surveys and Monographs (Amer. Math. Soc., Providence, 1995).

[8] R. Freese, J. Ježek and J. B. Nation, 'Lattices with large minimal extensions', preprint, 1997.

[9] J. Ježek and V. Slavík, 'Primitive lattices', Czechoslovak Math. J. 29 (1979), 595-634 Russian summary.

[10] K. M. Koh, 'On the Frattini sublattice of a lattice', Algebra Universalis 1 (1971), 104-116.

[11] R. McKenzie, 'Equational bases and non-modular lattice varieties', Trans. Amer. Math. Soc. 174 (1972), 1-43.

[12] J. B. Nation, 'An approach to lattice varieties of finite height', Algebra Universalis 27 (1990), 521-543.

[13] I. Rival, 'Maximal sublattices of finite distributive lattices', Proc. Amer. Math. Soc. 37 (1973), $417-420$.

Department of Mathematics and Computer Science

SUNY

New Paltz NY 12561

USA

e-mail: adamsm@matrix.newpaltz.edu

Department of Mathematics

University of Hawaii

Honolulu HI 96822

USA

e-mail: ralph@math.hawaii.edu jb@math.hawaii.edu
University of Bern

Sidlerstrasse 5

CH-3012 Bern

Switzerland

e-mail: schmid@math-stat.unibe.ch 